\title{
N, S-Codoped Carbon Shells Embedded with Ultrafine Co NPs for Reductive Amination with Formic Acid
}

Haotian Guo, ad Bowei Wang, abc* Pengzhi Qiu, a Ruixiao Gao, a Mingming Sun, a Ligong Chen, abc*

${ }^{a}$ School of Chemical Engineering and Technology, Tianjin University, Tianjin 300350, P. R. China.

b Collaborative Innovation Center of Chemical Science and Engineering (Tianjin), Tianjin 300072, P. R. China.

c Tianjin Engineering Research Center of Functional Fine Chemicals, Tianjin, P. R. China.

${ }^{d}$ Department of Chemistry, Emory University, Atlanta, Georgia 30322, United States.

* Corresponding author. E-mail: bwwang@tju.edu.cn (Bowei Wang);

1gchen@tju.edu.cn (Ligong Chen)

Number of pages: 10

Number of figures: 9

Number of Tables: 5 


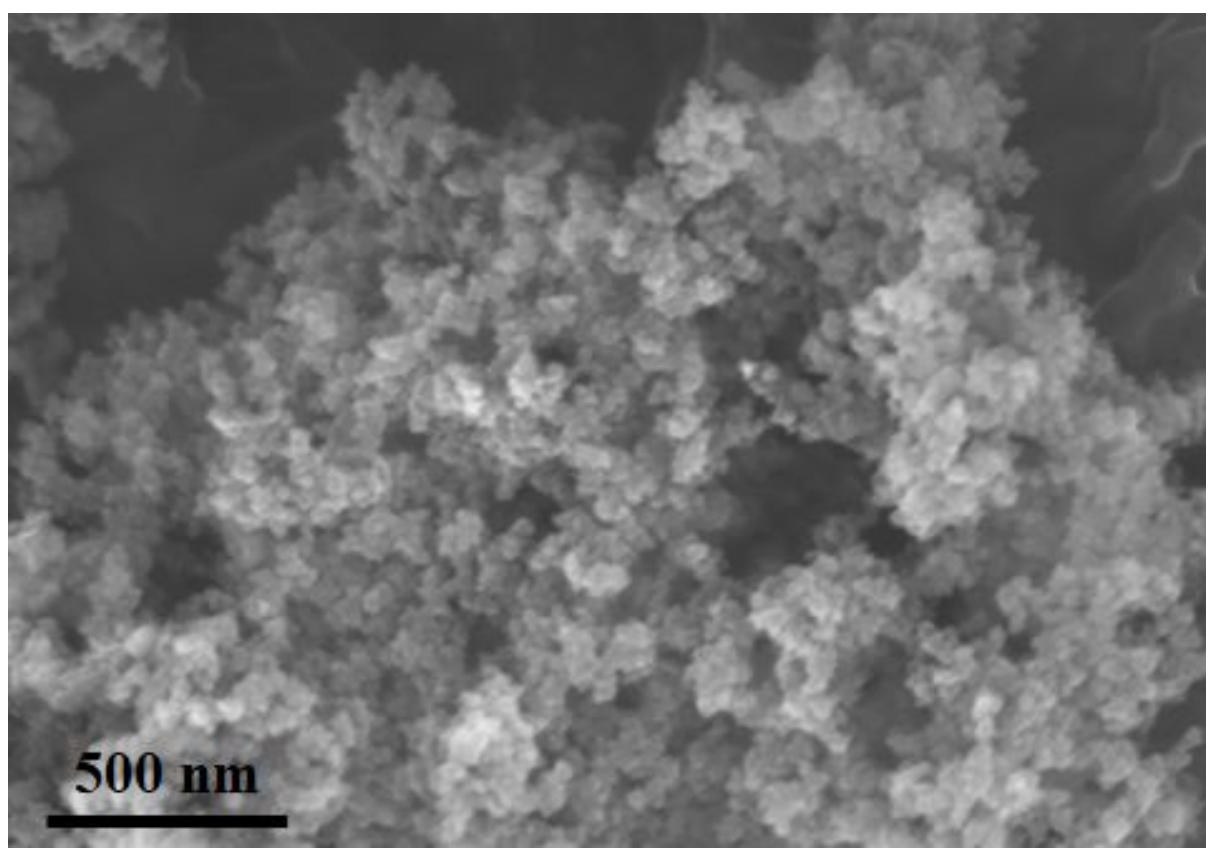

Fig. S1 The SEM image of SNW-1.

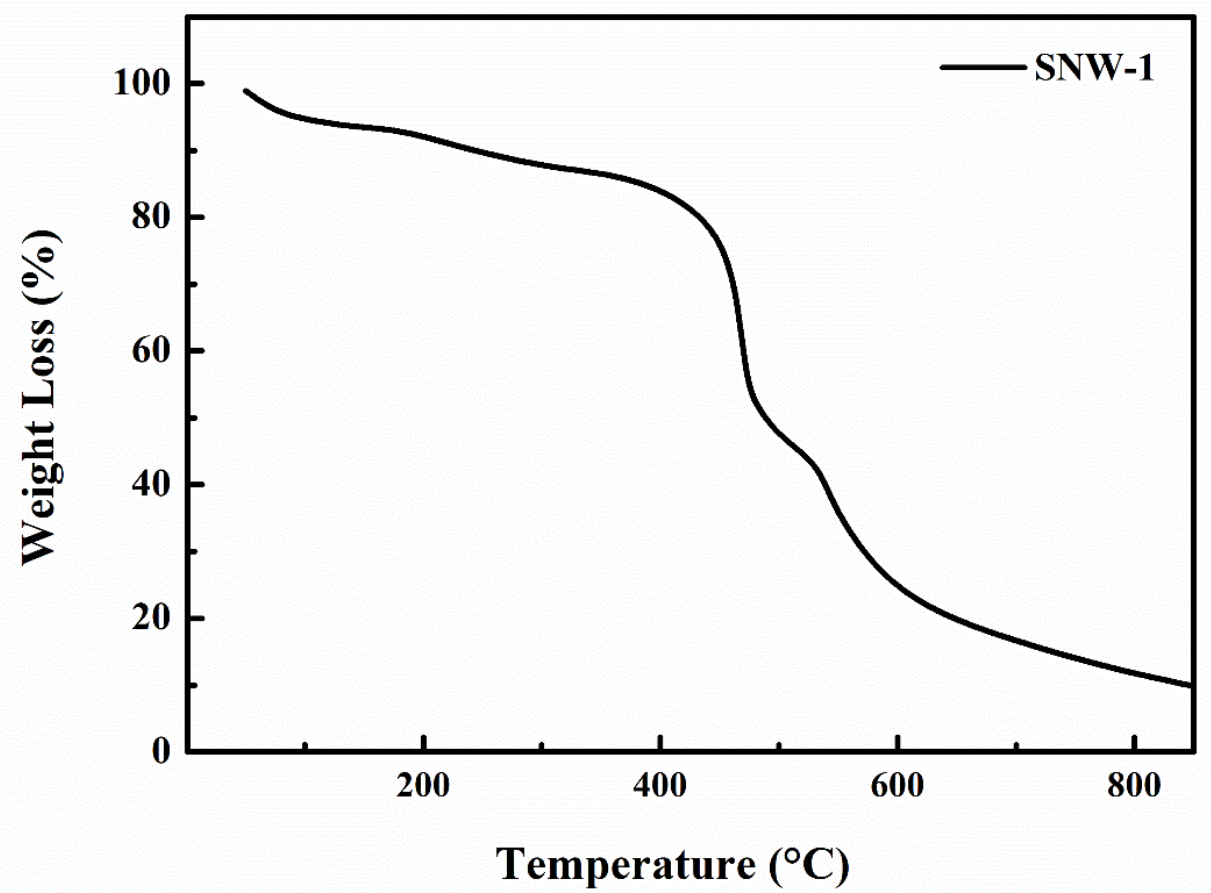

Fig. S2 The TGA curve of SNW-1. 

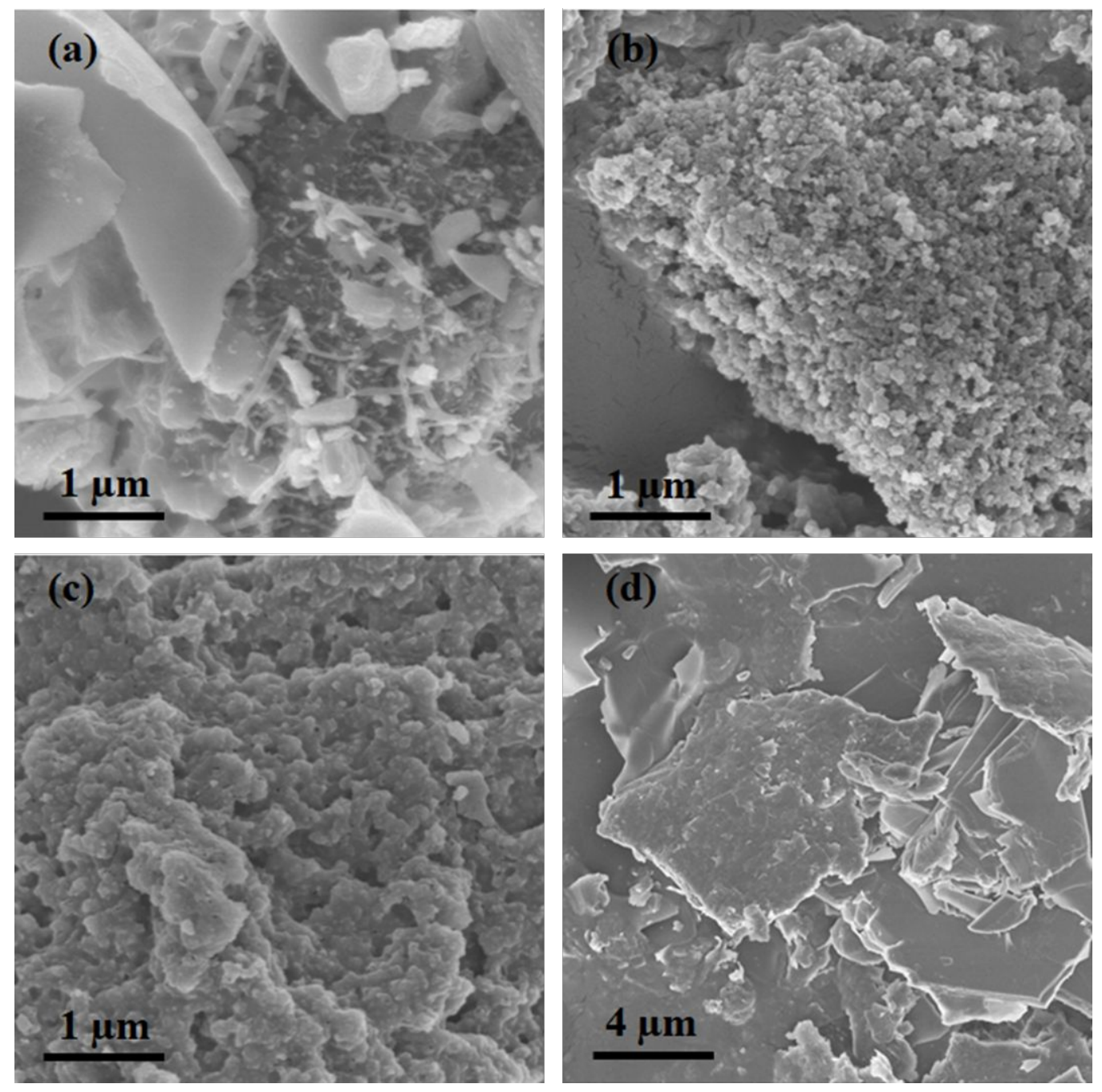

Fig. S3 The SEM images of Co@NSC-0 (a), Co@NSC-1 (b), Co@NSC-3 (c) and

Co@NSC-7 (d).
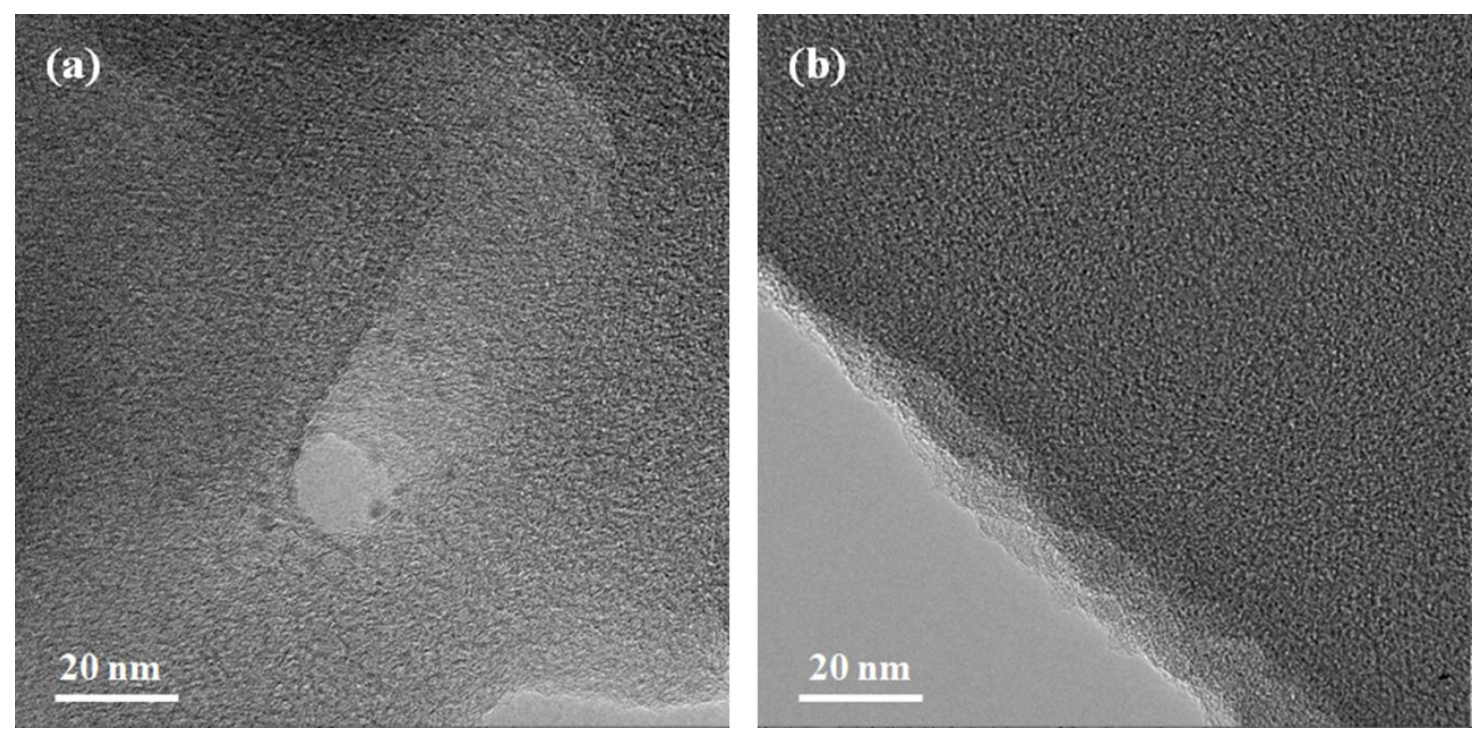
Fig. S4 The TEM images of Co@NSC-1 (a) and Co@NSC-7.

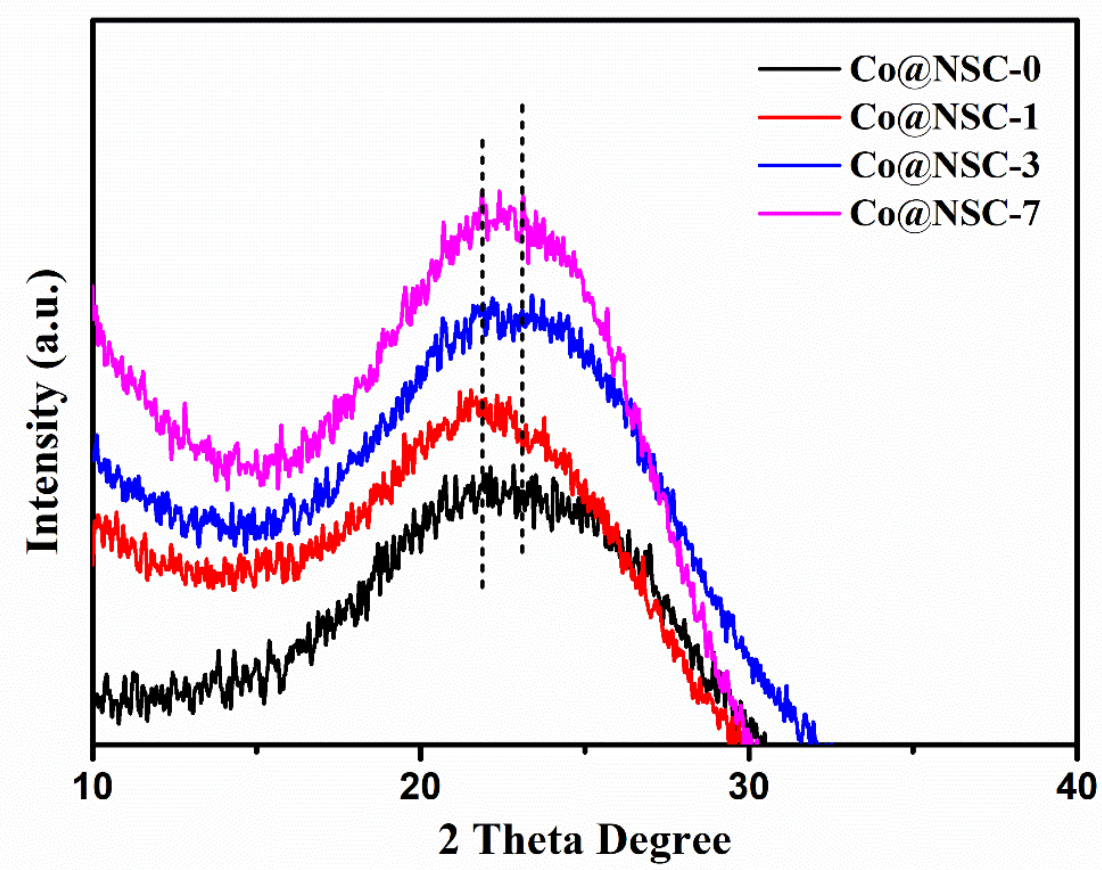

Fig. S5 The enlarged peak shift in XRD patterns of Co@NSC-X.

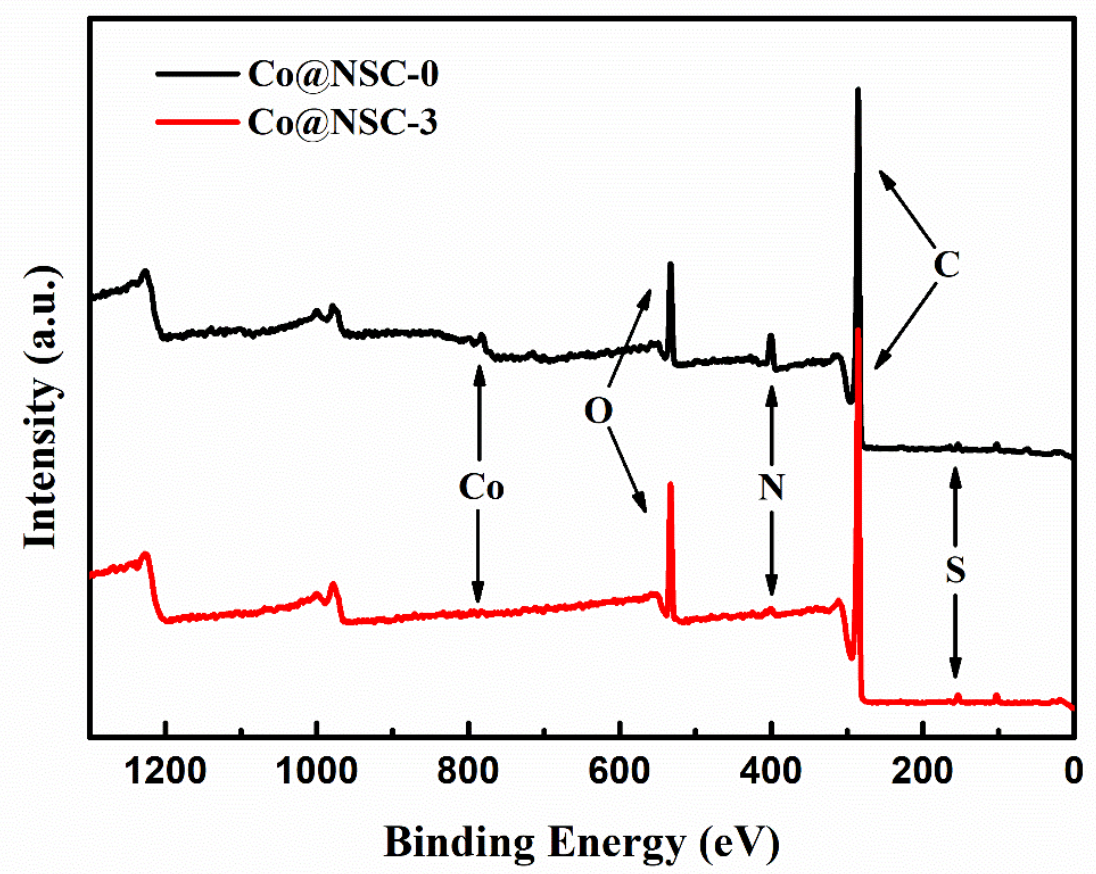


Fig. S6 The full survey spectra of Co@NSC-0 and Co@NSC-3.

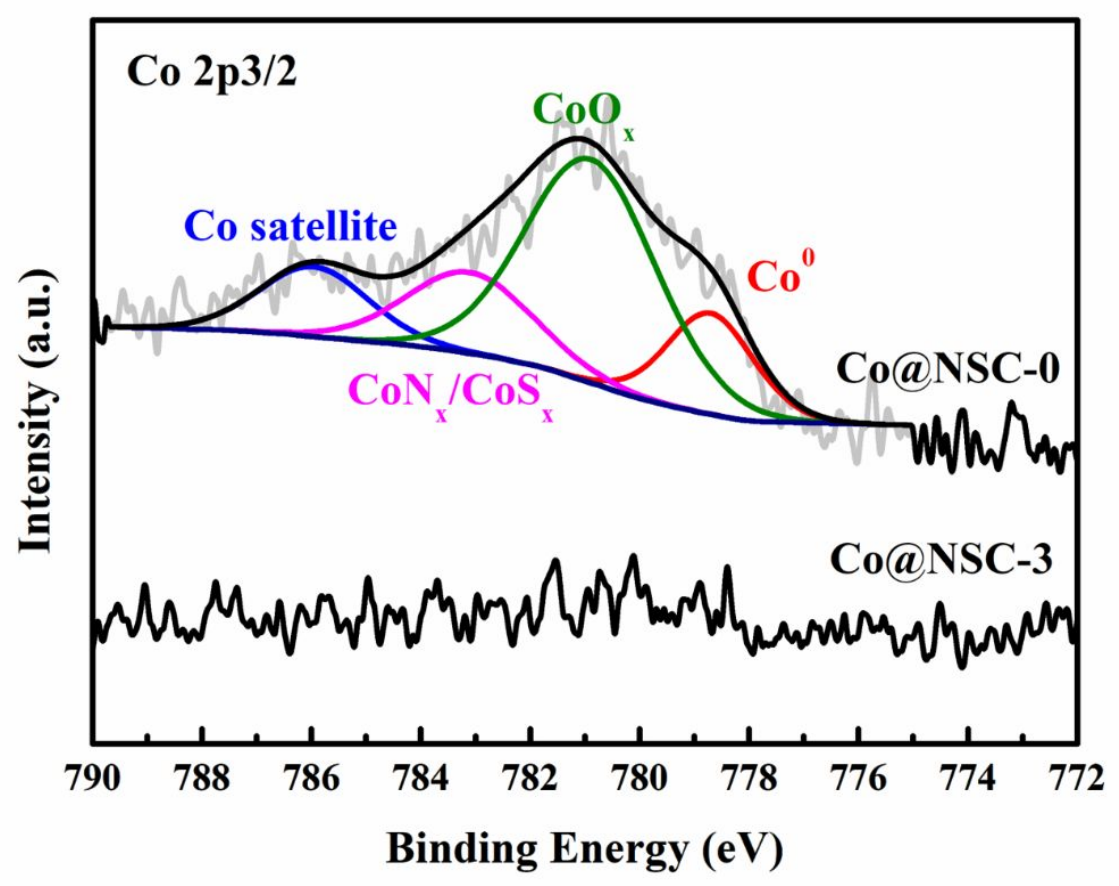

Fig. S7 The Co2p3/2 spectra of Co@NSC-0 and Co@NSC-3.

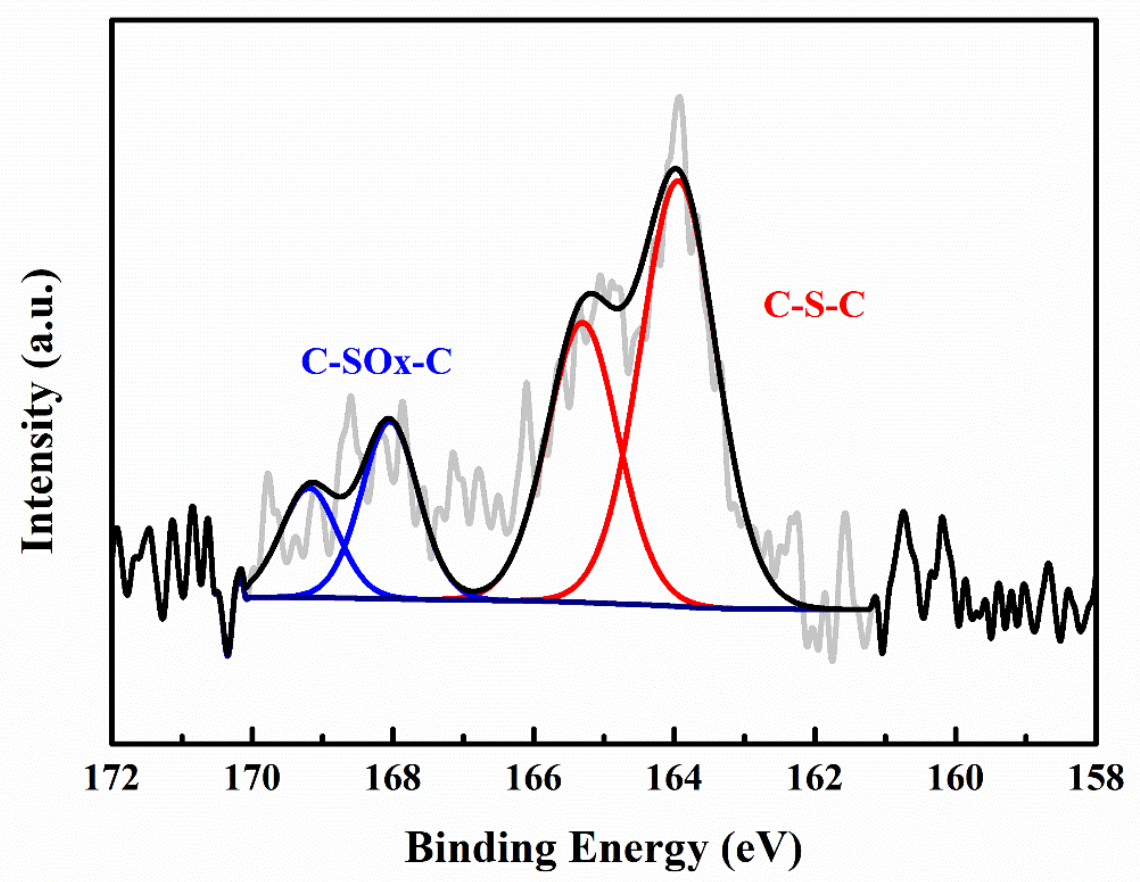


Fig. S8 The S2p spectrum of Co@NSC-1.

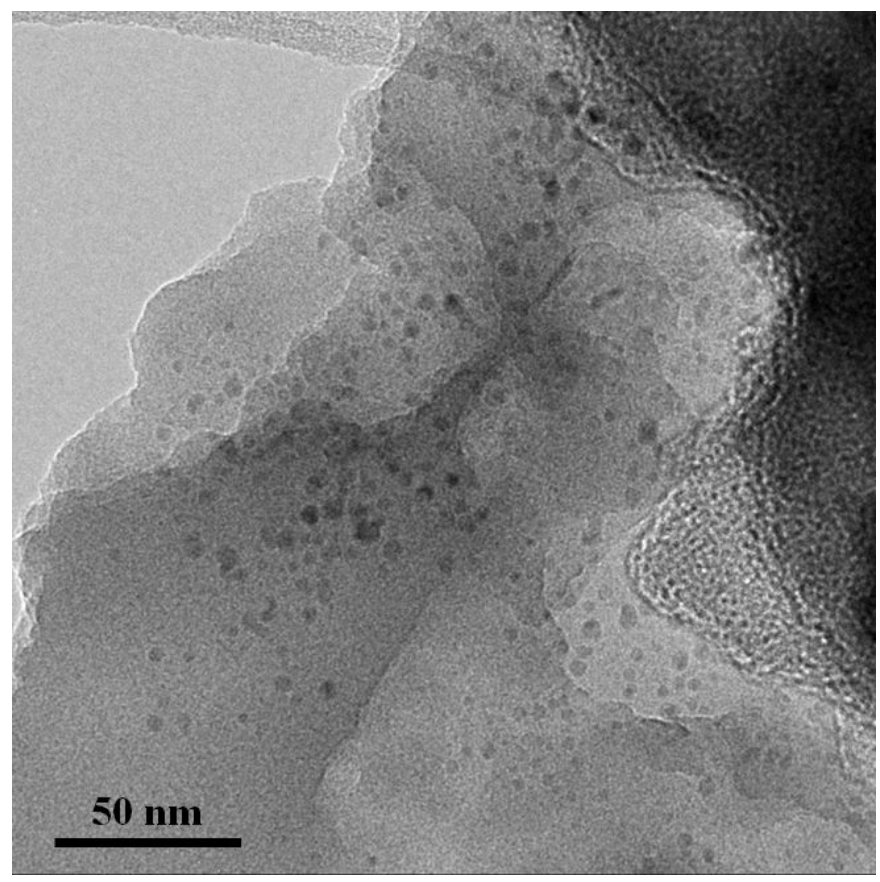

Fig. S9 The TEM image of used catalyst.

Table S1 The contents of N, S and Co in Co@NSC-x samples.

\begin{tabular}{cccccc}
\hline & $\mathrm{C}(\mathrm{wt} \%)^{\mathrm{a}}$ & $\mathrm{H}(\mathrm{wt} \%)^{\mathrm{a}}$ & $\mathrm{N}(\mathrm{wt} \%)^{\mathrm{a}}$ & $\mathrm{S}(\mathrm{wt} \%)^{\mathrm{a}}$ & $\mathrm{Co}(\mathrm{wt} \%)^{\mathrm{b}}$ \\
\hline SNW-1 & 37.31 & 4.30 & 43.71 & 3.00 & - \\
Co@NSC-0 & 60.58 & 1.57 & 9.63 & 1.27 & 5.71 \\
Co@NSC-1 & 67.98 & 1.72 & 5.08 & 1.14 & 2.02 \\
Co@NSC-3 & 69.01 & 2.02 & 3.42 & 0.85 & 1.14 \\
Co@NSC-7 & 72.98 & 1.83 & 3.92 & 0.40 & 0.52 \\
\hline
\end{tabular}

${ }^{\text {a }}$ detected by elemental analysis;

${ }^{\mathrm{b}}$ detected by ICP-OES. 
Table S2 The evaluation by CTH of nitrobenzene with formic acid ${ }^{\mathrm{a}}$

\begin{tabular}{cccc}
\hline & & & \\
\hline Entry & Catalyst & Conv. $(\%)$ & Sel.B $(\%)$ \\
\hline 1 & Co@NSC-0 & 38 & 100 \\
2 & Co@NSC-1 & 99 & 100 \\
3 & Co@NSC-3 & 97 & 100 \\
4 & Co@NSC-7 & 20 & 100 \\
\hline
\end{tabular}

${ }^{a}$ Reaction conditions: nitrobenzene $(0.5 \mathrm{mmol})$, solvent $(5 \mathrm{~mL})$, catalyst $(20 \mathrm{mg})$, formic acid $(1.5 \mathrm{mmol})$, time $(2 \mathrm{~h})$ and temperature $\left(110{ }^{\circ} \mathrm{C}\right)$; $\mathrm{GC}$ analysis using $\mathrm{n}$ dodecane as an internal standard;

Table S3 Comparison of the catalytic activity of Co@NSC-3 with recent reported Cobased catalysts for $\mathrm{CTH}$ of nitrobenzene.

\begin{tabular}{|c|c|c|c|c|c|c|c|c|}
\hline Entry & Catalyst & $\begin{array}{l}\text { FA } \\
\text { (eq) }\end{array}$ & $\begin{array}{c}\mathrm{T} \\
\left({ }^{\circ} \mathrm{C}\right)\end{array}$ & $\begin{array}{c}\mathrm{t} \\
\text { (h) }\end{array}$ & $\begin{array}{c}\mathrm{Co} \\
(\mathrm{wt} \%)\end{array}$ & $\begin{array}{l}Y_{\mathrm{AN}^{\mathrm{a}}} \\
(\%)\end{array}$ & $\begin{array}{l}\mathrm{CP}^{\mathrm{b}} \\
\left(\mathrm{h}^{-1}\right)\end{array}$ & Ref. \\
\hline 1 & $\mathrm{Co}_{3} \mathrm{O}_{4}-\mathrm{NGr} @ \mathrm{C}$ & 3.5 & 100 & 15 & 3.05 & 96 & 3.20 & {$[1]$} \\
\hline 2 & Co@CN-800 & 3 & 110 & 10 & 23.9 & 100 & 0.62 & {$[2]$} \\
\hline 3 & Co@NC-600 & 9.78 & 90 & 6 & 9.3 & 99.9 & 1.32 & {$[3]$} \\
\hline 4 & Co@NPC-800 & 4 & 120 & 24 & 3.54 & 90 & 0.69 & {$[4]$} \\
\hline 5 & Co-NSPC-850 & 3 & 110 & 3 & 10.02 & 99 & 4.85 & {$[5]$} \\
\hline 6 & Co-Nx/C-800-AT & 3 & 110 & 12 & 0.25 & $>99$ & 48.59 & {$[6]$} \\
\hline 7 & Co@NSC-3 & 3 & 110 & 3 & 1.12 & 97 & 41.76 & $\begin{array}{l}\text { This } \\
\text { work }\end{array}$ \\
\hline
\end{tabular}

${ }^{\mathrm{a}} \mathrm{Y}_{\mathrm{AN}}$ means yield of aniline. 
b CP means catalyst productivity, which is calculated by moles of nitrobenzene consumed divided by total moles of cobalt per hour.

Table S4 The solvent selection for one-pot hydrogenation and reductive amination ${ }^{\mathrm{a}}$.

\begin{tabular}{|c|c|c|c|c|c|c|}
\hline No & po & & $\underset{d}{\mathbf{N H}_{2}}$ & $H_{N}$ & $\mathbf{E}$ & FN \\
\hline Entry & Solvent & Conv.A(\%) & Sel.C $(\%)$ & Sel.D $(\%)$ & Sel.E(\%) & Sel.F(\%) \\
\hline 1 & toluene & 94.8 & 10.7 & 0.9 & 74.9 & 13.5 \\
\hline 2 & THF & 79.7 & 3.0 & 1.3 & 67.2 & 28.5 \\
\hline 3 & 1,4-Dioxane & 8.9 & 2.0 & 19.9 & 77.2 & 0.9 \\
\hline 4 & n-Hexane & 28.5 & 3.9 & 0.0 & 87.8 & 8.3 \\
\hline 5 & isopropanol & 75.9 & 3.4 & 0.4 & 65.9 & 30.3 \\
\hline 6 & DMF & 92.8 & 43.1 & 0.6 & 17.2 & 39.1 \\
\hline 7 & $\mathrm{H}_{2} \mathrm{O}$ & 95.2 & 48.2 & 0 & 17.0 & 34.8 \\
\hline 8 & $\mathrm{THF} / \mathrm{H}_{2} \mathrm{O}^{\mathrm{b}}$ & 100 & 25.1 & 2.5 & 18.5 & 53.9 \\
\hline
\end{tabular}

a Reaction conditions: nitrobenzene (0.5 mmol), benzaldehyde (1 mmol), Co@NSC-3 (20 mg), solvent $(5 \mathrm{~mL})$, formic acid $(2 \mathrm{mmol})$ and $150{ }^{\circ} \mathrm{C}$; Conversion and the selectivity were calculated based on nitro compounds, and GC analysis using ndodecane as an internal standard. 
${ }^{\mathrm{b}} \mathrm{V}_{\mathrm{THF}} / \mathrm{V}_{\mathrm{H} 2 \mathrm{O}}=10: 1$

Table S5 The optimization of one-pot hydrogenation and reductive amination ${ }^{\mathrm{a}}$.

\begin{tabular}{|c|c|c|c|c|c|c|c|}
\hline No & + & FO & & $\underset{\mathrm{N}}{\mathbf{N H}_{2}}$ & 少 & $\mathbf{E}$ & Hi \\
\hline Entry & $\mathrm{T}$ & FA(eq.) & Conv.A(\%) & Sel.C(\%) & Sel.D $(\%)$ & Sel.E(\%) & Sel.F(\%) \\
\hline 1 & 110 & 4 & 100 & 33.4 & 3.5 & 54.3 & 8.8 \\
\hline 2 & 130 & 4 & 100 & 19.9 & 2.6 & 40.1 & 37.4 \\
\hline 3 & 150 & 4 & 100 & 25.1 & 2.5 & 18.5 & 53.9 \\
\hline 4 & 170 & 4 & 100 & 26.5 & 0.7 & 9.3 & 63.4 \\
\hline 5 & 170 & 5 & 100 & 1.4 & 2.0 & 7.0 & 89.6 \\
\hline 6 & 170 & 6 & 100 & 3.3 & 1.0 & 0.7 & 94.9 \\
\hline
\end{tabular}

${ }^{a}$ Reaction conditions: nitrobenzene $(0.5 \mathrm{mmol})$, benzaldehyde (1 mmol), Co@NSC-3 $(20 \mathrm{mg}), \mathrm{THF} / \mathrm{H}_{2} \mathrm{O}\left(\mathrm{V}_{\mathrm{THF}} / \mathrm{V}_{\mathrm{H} 2 \mathrm{O}}=10: 1,5 \mathrm{~mL}\right)$; Conversion and selectivity were calculated based on nitro compounds, and GC analysis using n-dodecane as an internal standard. 


\section{Reference}

[1] R. V. Jagadeesh, D. Banerjee, P. B. Arockiam, H. Junge, K. Junge, M. M. Pohl, J. Radnik, A. Bruckner, M. Beller Green Chemistry. 2015, 17, 898-902.

[2] P. Zhou, Z. H. Zhang, L. Jiang, C. L. Yu, K. L. Lv, J. Sun, S. G. Wang Applied Catalysis BEnvironmental. 2017, 210, 522-532.

[3] M. Yuan, Y. Long, J. Yang, X. W. Hu, D. Xu, Y. Y. Zhu, Z. P. Dong Chemsuschem. 2018, $11,4156-4165$

[4] Y. N. Duan, T. Song, X. S. Dong, Y. Yang Green Chemistry. 2018, 20, 2821-2828.

[5] H. Guo, R. Gao, M. Sun, H. Guo, B. Wang, L. Chen ChemSusChem. 2019, 12, 487-494.

[6] P. Zhou, L. Jiang, F. Wang, K. J. Deng, K. L. Lv, Z. H. Zhang Science Advances. 2017, 3. 